\title{
DILATOMETRIC STUDY OF THE PHASE TRANSITION IN $\left(\mathrm{CH}_{3} \mathrm{NH}_{3}\right)_{5} \mathrm{Bi}_{2} \mathrm{Cl}_{11}$
}

\author{
H. PYKaCZ \\ Institute of Physics, Technical University of Wrocław \\ Wybrzeże Wyspiańskiego 27, 50-370 Wrocław, Poland
}

AND R. JAKUBAS

Institute of Chemistry, University of Wroclaw

F. Joliot-Curie 14, 50-383 Wrocław, Poland

(Received December 11, 1990)

The paper reports a dilatometric study on $\left(\mathrm{CH}_{3} \mathrm{NH}_{3}\right)_{5} \mathrm{Bi}_{2} \mathrm{Cl}_{11}$ single crystals. It is shown that elongation of the crystal is continuous but linear thermal expansion coefficients are discontinuous at phase transition temperature'.

PACS numbers: $64.70 . \mathrm{Kb}, 65.70 .+\mathrm{y}$

Recently a new crystal was grown: namely $\left(\mathrm{CH}_{3} \mathrm{NI}_{3}\right)_{5} \mathrm{Bi}_{2} \mathrm{Cl}_{11}$ with promising dielectric properties [1]. This compound crystallizes at room temperature in orthorombic symmetry with space group $P c a 2_{1}$. With increasing temperature crystals transform from the ferroelectric phase to the paraelectric phase at about $T_{\mathrm{C}}=308 \mathrm{~K}$. The phase transition is the second order with a peak of electric permittivity $5 \times 10^{3}$ [1]. The authors of Ref. [2] paid attention to similarity of the dielectric properties of this crystal and isomorphic $\left(\mathrm{CH}_{3} \mathrm{NI}_{3}\right)_{5} \mathrm{Bi}_{2} \mathrm{Br}_{11}$ crystal, for which dilatometric anomaly was shown in [3].

In this short note we report results of dilatometric measurements performed to study the ferroelectric phase transition in $\left(\mathrm{CII}_{3} \mathrm{NII}_{3}\right)_{5} \mathrm{Bi}_{2} \mathrm{Cl}_{11}$. Single crystals were prepared as described in [1]. A capacitive quartz dilatometer was used to measure the elongation of bars $9.2 \mathrm{~mm}$ long. The capacitance was measured with an automatic $C$-bridge versus temperature on heating with a constant rate of $4.35 \times 10^{-3} \mathrm{~K} \mathrm{~s}^{-1}$ and also at constant temperatures. The thermal dilatations in the $a, b$ and $c$ directions and respective linear thermal expansion coefficients: $\alpha_{a}, \alpha_{b}$ and $\alpha_{c}$, are shown in Figs. 1-3. It was assumed that $\Delta l=0$ at $295 \mathrm{~K}$. Anomalous changes of these quantities are observed in the vicinity of $T_{\mathrm{C}}=308 \mathrm{~K}$. At the transition point continuous dilatations in all directions are seen on heating, 


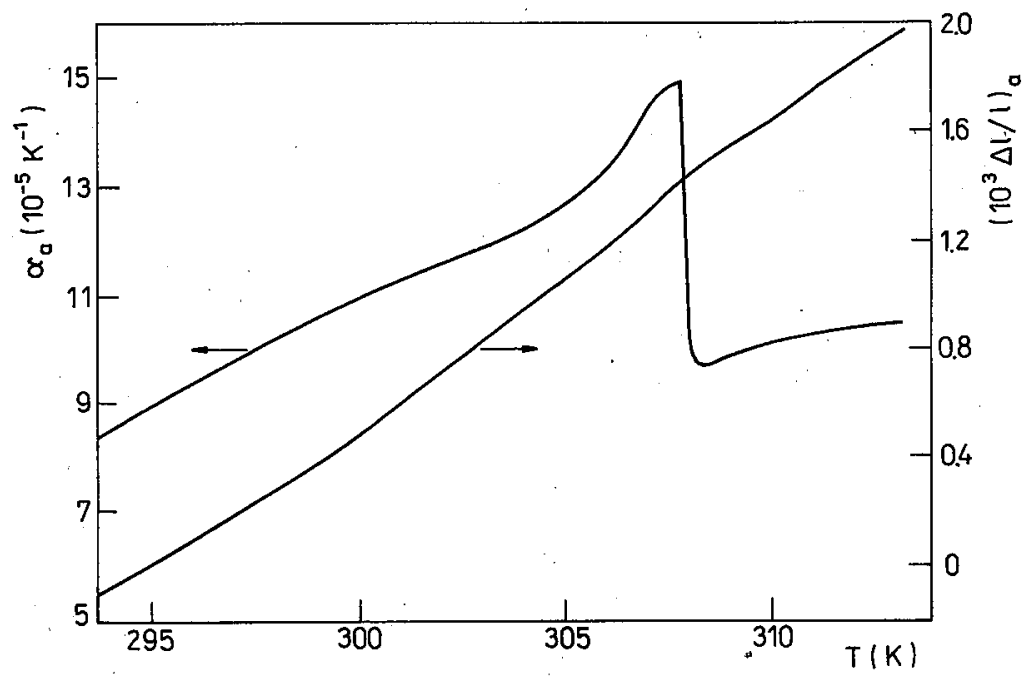

Fig. 1. Elongation of the crystal measured relative to the length at $295 \mathrm{~K}$ and linear thermal expansion coefficient along $a$-axis.

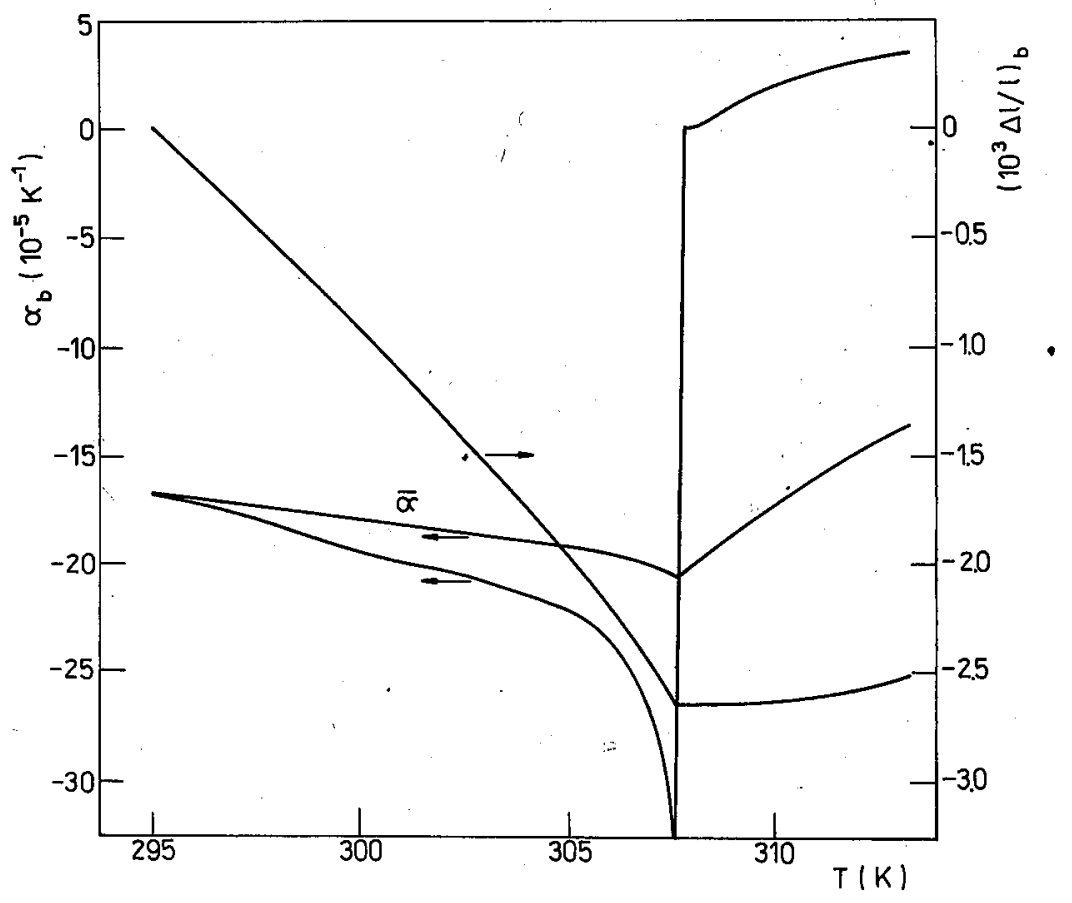

Fig. 2. Dilatation of the crystal along $b$-axis. $\bar{\alpha}$ is the mean value of the expansion coefficient between $T$ and $295 \mathrm{~K}$. 
whereas the thermal expansion coefficients are changing discontinuously. These results speak in favour of the second order phase transition. The thermal expansion coefficient along the $a$-axis $\alpha_{a}$ (Fig. 1 ) is positive and increases with temperature in the ferroelectric phase, while the one along the $b$-axis having negative value in the polar phase, changes its sign exactly at $T_{\mathrm{C}}$ and becomes positive afterwards (Fig. 2). In Fig. 2 the mean value of the thermal expansion coefficient $\bar{\alpha}$ defined

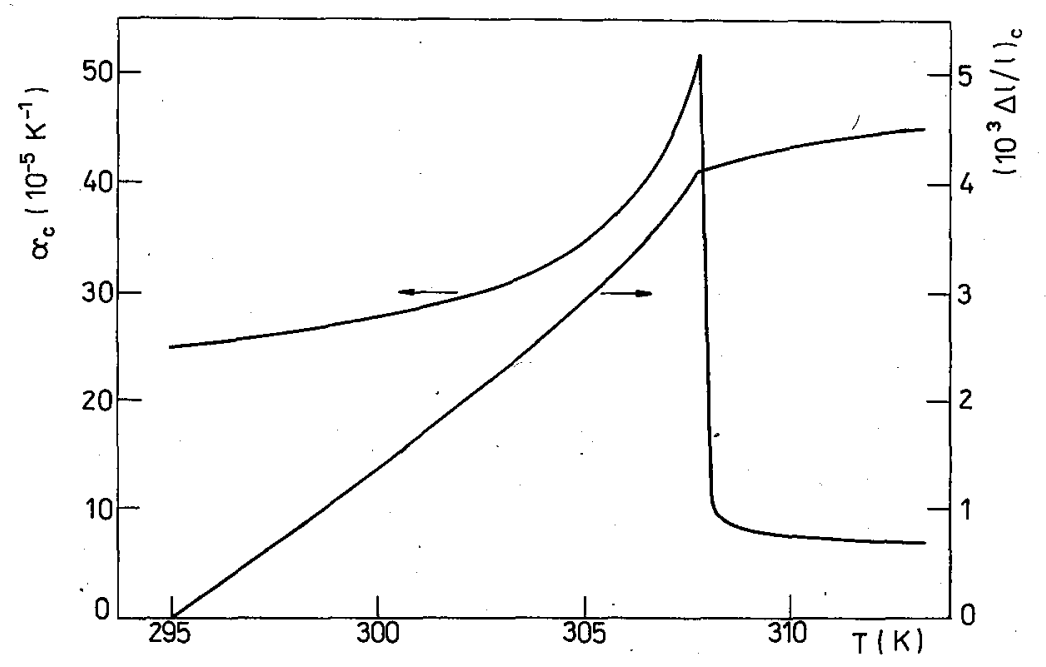

Fig. 3. Dilatation of the crystal along the c-axis.

by $\left(l_{T}-l_{295}\right) / l_{295}(T-295 \mathrm{~K})$ is also shown. It should be noticed that the $\alpha_{\mathrm{C}}$ is relatively big, and about three times larger than the one for the $a$-axis (Fig. $3)$. $\left(\mathrm{CH}_{3} \mathrm{NII}_{3}\right)_{5} \mathrm{Bi}_{2} \mathrm{Cl}_{11}$ single crystals possess very large thermal expansion especially over the temperature range from room temperature up to the transition point. The volume thermal expansion coefficient increases with temperature from $17 \times 10^{-5} \mathrm{~K}^{-1}$ at $295 \mathrm{~K}$ to $30 \times 10^{-5} \mathrm{~K}^{-1}$ at $T_{\mathrm{C}}$. The dilatometric measurements indicate that the symmetry of the crystal both in ferroelectric and paraclectric phases is not higher than orthorhombic. The phase transition then can be described by $m m m F m m 2$. Comparing the obtained dilatometric results with those for $\left(\mathrm{CII}_{3} \mathrm{NII}_{3}\right)_{5} \mathrm{Bi}_{2} \mathrm{Br}_{11}[3]$ one can see large similarity of the dilatometric propertics. Crystals containing $\mathrm{Cl}$ have slightly greater thermal expansion coefficients and their phase transition temperature is lower by $3 \mathrm{~K}$.

\section{References}

[1] R. Jakubas, L. Sobczyk, J. Lefebvre, Ferroelectrics 100, 143 (1980).

[2] J. Mróz, R. Jakubas, Ferroelectr. Lell. Sect. 11, 53 (1990).

[3] R. Jakubas, II. Pykacz, Phys. Slalus Solidi A 115, K17. (1989). 